\title{
Predictive value of the QFR in detecting vulnerable plaques in non-flow limiting lesions: a combined analysis of the PROSPECT and IBIS-4 study
}

\author{
Hannah Safi, ${ }^{1,2}$ Christos V. Bourantas ${ }^{1,2,3}$. Anantharaman Ramasamy ${ }^{1,3} \cdot$ Thomas Zanchin $^{1,4} \cdot$ Sarah Bär ${ }^{4}$. \\ Vincenzo Tufaro $^{1}$. Chongying Jin ${ }^{1,5} \cdot$ Ryo Torii $^{6}$. Alexios Karagiannis ${ }^{7}$. Johan H. C. Reiber ${ }^{8}$. Anthony Mathur ${ }^{1,3}$. \\ Yoshinubo Onuma ${ }^{9} \cdot$ Stephan Windecker ${ }^{4} \cdot$ Alexandra Lansky $^{2,10} \cdot$ Akiko Maehara $^{11} \cdot$ Patrick W. Serruys $^{12}$. \\ Peter Stone $^{13} \cdot$ Andreas Baumbach $^{1,3} \cdot$ Gregg W. Stone $^{11} \cdot$ Lorenz Räber $^{4}$
}

Received: 10 December 2019 / Accepted: 22 February 2020

(c) Springer Nature B.V. 2020

\begin{abstract}
Studies have shown that the quantitative flow ratio (QFR), recently introduced to assess lesion severity from coronary angiography, provides useful prognostic information; however the additive value of this technique over intravascular imaging in detecting lesions that are likely to cause events is yet unclear. We analysed data acquired in the PROSPECT and IBIS-4 studies, in particular the baseline virtual histology-intravascular ultrasound (VH-IVUS) and angiographic data from 17 non-culprit lesions with a presumable vulnerable phenotype (i.e., thin or thick cap fibroatheroma) that caused major adverse cardiac events or required revascularization (MACE) at 5-year follow-up and from a group of 78 vulnerable plaques that remained quiescent. The segments studied by VH-IVUS were identified in coronary angiography and the QFR was estimated. The additive value of 3-dimensional quantitative coronary angiography (3D-QCA) and of the QFR in predicting MACE at 5 year follow-up beyond plaque characteristics was examined. It was found that MACE lesions had a greater plaque burden (PB) and smaller minimum lumen area (MLA) on VH-IVUS, a longer length and a smaller minimum lumen diameter (MLD) on 3D-QCA and a lower QFR compared with lesions that remained quiescent. By univariate analysis MLA, PB, MLD, lesion length on 3D-QCA and QFR were predictors of MACE. In multivariate analysis a low but normal QFR $(>0.80$ to $<0.97)$ was the only independent prediction of MACE (HR 3.53, 95\% CI 1.16-10.75; P=0.027). In non-flow limiting lesions with a vulnerable phenotype, QFR may provide additional prognostic information beyond plaque morphology for predicting MACE throughout 5 years.
\end{abstract}

Keywords Vulnerable plaque $\cdot$ Quantitative coronary angiography $\cdot$ QFR

Christos V. Bourantas

cbourantas@gmail.com

1 Department of Cardiology, Barts Heart Centre, Barts Health NHS Trust, London, UK

2 Institute of Cardiovascular Sciences, University College London, London, UK

3 William Harvey Research Institute, Queen Mary University London, London, UK

4 Department of Cardiology, Bern University Hospital, Bern, Switzerland

5 Department of Cardiology, Sir Run Run Shaw Hospital, School of Medicine, Zhejiang University, Hangzhou, China

6 Department of Mechanical Engineering, University College London, London, UK
7 CTU Bern, Institute of Social and Preventive Medicine, Bern University, Bern, Switzerland

8 Medis Medical Imaging Systems bv, Leiden, The Netherlands

9 Department of Interventional Cardiology, Thoraxcenter, Erasmus Medical Center, Rotterdam, The Netherlands

10 Division of Cardiovascular Medicine, Department of Internal Medicine, Yale School of Medicine, New Haven, CT, USA

11 Department of Cardiology, Columbia University Medical Center and the Cardiovascular Research Foundation, New York, NY, USA

12 Faculty of Medicine, National Heart \& Lung Institute, Imperial College London, London, UK

13 Cardiovascular Division, Brigham \& Women's Hospital, Harvard Medical School, Boston, MA, USA 


\section{Introduction}

Fractional flow reserve (FFR) is the gold standard for assessing the physiologic significance of a coronary artery lesion and planning treatment. This modality, which also appears able to provide useful prognostic information even in non-flow limiting lesions [1], involves advancement of a pressure wire across the lesion and adenosine administration to achieve hyperaemia and thus it comes with additional procedure-related risks of adverse events, extended procedure time and increased cost [2]. Quantitative flow ratio (QFR), a wire-free, hyperaemia-free method has been recently introduced to overcome these limitations and derive FFR from models reconstructed from 3-dimensional quantitative coronary angiography (3D-QCA) [3]. Recent clinical studies have supported the potential clinical value of QFR showing that it enables not only accurate detection of flow limiting lesions but also identification of patients at risk from cardiovascular events [4-7].

Cumulative data has shown that imaging-derived variables combined with computationally-derived physiological indices enables more accurate detection of lesions that are likely to progress and cause events. In the EMERLAD study, plaque characteristics and the FFR estimated by computed tomography allowed detection of lesions that caused myocardial infarction with a higher accuracy than plaque morphology (c-index: 0.789 vs. $0.747 ; \mathrm{P}=0.014$ ) [8]. However, no study today has assessed whether computationallyderived FFR - using the QFR software - has additive predictive value compared with intravascular imaging alone.

\section{Methods}

\section{Study design}

The present study is a post hoc analysis of data obtained from the Intergraded Biomarkers Imaging Study 4 (IBIS-4) and the Providing Regional Observations to Study Predictors of Events in the Coronary Tree (PROSPECT) clinical studies. The study design of these trials, the definitions and endpoints as well as the inclusion and exclusion criteria have been described in detail elsewhere $[9,10]$. In summary, the IBIS-4 trial was a prospective multi-modality multicenter study which aimed to investigate the effect of aggressive statin therapy on plaque composition and burden in patients admitted with a ST-elevation myocardial infarction (STEMI). All included patients $(n=103)$ had successful revascularization and 3-vessel virtual histology intravascular ultrasound (VH-IVUS) and optical coherence tomography (OCT) imaging at baseline and 13-month follow-up.
The PROSPECT study was a prospective large-scale invasive imaging study that aimed to examine the ability of VH-IVUS in detecting vulnerable plaques that would evolve and cause events. The study included 697 patients admitted with an acute coronary syndrome that had 3-vessel VH-IVUS imaging of the proximal $60-80 \mathrm{~mm}$ segment and successful percutaneous coronary intervention (PCI) in all the flow limiting lesions.

The patients recruited in the IBIS-4 study were followed-up for a median of 5 years whereas those in the PROSPECT trial for 3.4 years. The clinical, angiographic and intravascular imaging data in both studies allowed detection of untreated lesions that were assessed by VH-IVUS at baseline and caused cardiovascular events (defined as cardiac death, myocardial infarction, or revascularization because of progressive or unstable angina). In addition, in the IBIS-4 study lesions that exhibited disease progression and a significant stenosis (\% diameter stenosis, DS $\geq 70 \%$ on QCA) or an intermediate stenosis $(70 \% \geq \% \mathrm{DS} \geq 50 \%)$ associated with recurrent angina or evidence of ischemia during exercise test at 13 months with follow-up angiography were considered culprit and underwent revascularization. The primary endpoints of the study were lesions assessed by VH-IVUS at baseline that either progressed and required revascularization on repeat angiography, or lesions that caused cardiac death, myocardial infarction, or revascularization because of progressive or unstable angina (MACE).

In the IBIS-4 study all the lesions associated with MACE had either a thin (TCFA, $n=13$ ) or a thick-cap fibroatheroma (ThCFA, $n=2$ ) phenotype. In the PROSPECT study 43 of the lesions that were studied by VHIVUS at baseline and caused events had a fibroatheroma phenotype (TCFA, $n=25$; ThCFA, $n=18$ ), 7 had a nonfibroatheroma phenotype and 4 were unclassified lesions $[9,10]$. From these 43 lesions, we analysed only data from the lesions that were included in the PROSPECT endothelial shear stress (ESS) sub-study, a study that aimed to investigate the prognostic value of ESS in predicting events [11]. The PROSPECT ESS sub-study included lesions where matching of VH-IVUS and X-ray angiography was feasible and lesions that had a length $\geq 9 \mathrm{~mm}$ and a vulnerable phenotype (TCFA, ThCFA). In addition, we included a control group of lesions with a vulnerable phenotype that remained quiescent from: (1) the PROSPECT ESS sub-study and from (2) the patients recruited in the IBIS-4 study in Bern University Hospital. Lesions with suboptimal angiographic images (for instance vessel overlapping or foreshortening or poor opacification of the lumen silhouette) and cases where the DICOM file did not include all the required information for QFR analysis were excluded from the study. 


\section{VH-IVUS analysis}

VH-IVUS analysis was carried out by independent core-laboratories (Cardialysis B.V., Rotterdam, The Netherlands in IBIS-4 and Cardiovascular Research Foundation, New York, NY in PROSPECT) using dedicated software (QIvus, Medis, Leiden, The Netherlands for the IBIS-4 study and QCUCMS, Medis, Leiden, the Netherlands for the PROSPECT study). In the IBIS-4 study, analysis was performed only for the segment that was evaluated by VH-IVUS at baseline and follow-up, whereas in the PROSPECT study the analysis was carried out for the entire imaged coronary artery.

VH-IVUS segmentation was carried out at every enddiastolic frame; in each frame the lumen and external elastic membrane (EEM) area were annotated and their dimensions, the plaque area and burden (PB) and its composition (fibrotic, fibrofatty, calcific and necrotic core area and burden) were estimated. In the studied segments, lesions were characterised as $\geq 3$ consecutive VH-IVUS frames with $\mathrm{PB} \geq 40 \%$; in each lesion the plaque composition was used to classify it to one of the following phenotypes: pathologic intimal thickening, fibrotic, fibrocalcific, TCFA and ThCFA [12].

\section{D-QCA reconstruction}

Anatomical landmarks such as side branches observed in X-ray angiography and VH-IVUS were utilised to determine the distal and proximal ends of the segment that was evaluated by VH-IVUS on coronary angiography. Two enddiastolic angiographic projections that were more than $25^{\circ}$ apart, where there was no foreshortening or overlapping of the segment of interest and permitted accurate delineation of the lumen silhouette were chosen to reconstruct its anatomy. This was carried out using an established and well-validated software (QAngio XA 3D RE, Medis, Leiden, the Netherlands) [13]. In the obtained geometries the lesion length, DS and minimum lumen diameter (MLD) were estimated.

\section{QFR analysis}

QFR was computed using the Medis Suite XA/QAngio XA 3D/QFR software (Medis, Leiden, the Netherlands). QFR was computed using two approaches: (1) assuming a fixed blood flow (fQFR) and (2) taking into account the flow velocity estimated by the time needed for the contrast agent to fill the segment of interest which is known as contrastflow QFR (cQFR) [3].

\section{Statistical methods}

Numerical variables are presented as median and interquartile ranges while categorical variables as absolute values and percentages. Comparison between numerical variables were performed using the Mann-Whitney U test while categorical variables were compared using the chi-square test. Cox regression analysis was used to identify VH-IVUS, 3D-QCA-derived and QFR predictors associated with MACE. For the 3D-QCA and QFR variables associated with MACE $(\mathrm{P}<0.05)$ receiver-operating characteristics $(\mathrm{ROC})$ curve analysis was performed to identify the best cut-off that predicted MACE. The variables with the highest area under the curve (AUC) that were not co-linear $(r>0.5)$ were entered into a multivariable model to identify independent predictors of MACE.

VH-IVUS and QFR variables were used to classify the lesions into groups. Kaplan-Meier plots were used to display time to event; comparison of MACE rate between groups with different plaque characteristics and QFR values was performed using the log-rank test. Analyses were performed in Stata (version 15.1, StataCorp LP, College Station, TX) and SPSS (version 23; IBM Corp, Armonk, NY, USA). A $\mathrm{P}$-value $<0.05$ was considered statistically significant.

\section{Results}

\section{Patient demographics}

QFR analysis was possible in 17 MACE lesions with a thin or thick cap fibroatheroma phenotype (5 from the PROSPECT ESS study and 12 from the IBIS-4 study) (Fig. 1). From the 122 lesions that were included in the PROSPECT ESS study and did not cause events, 8 were excluded from the present analysis because of suboptimal angiographic views and 102 were excluded because of insufficient information in the DICOM file for QFR analysis. From the 78 thin or thick cap fibroatheromas that were recruited in the IBIS-4 study from Bern University Hospital, 12 lesions were excluded from the present analysis because of suboptimal angiographic views. Therefore the final analysis included 17 MACE lesions and a control group of 78 lesions that remained quiescent.

The baseline characteristics of the patients $(n=60)$ included in the present analysis are shown in Table 1. There were no differences in the baseline demographics between patients who had a MACE and those that did not have an event.

\section{Lesions characteristics}

Table 2 shows the morphological and angiographic variables as well as the QFR values in the studied lesions. All the studied lesions were non-flow limiting by QFR (range: 0.81-1.00). 


\section{IBIS 4 Study}

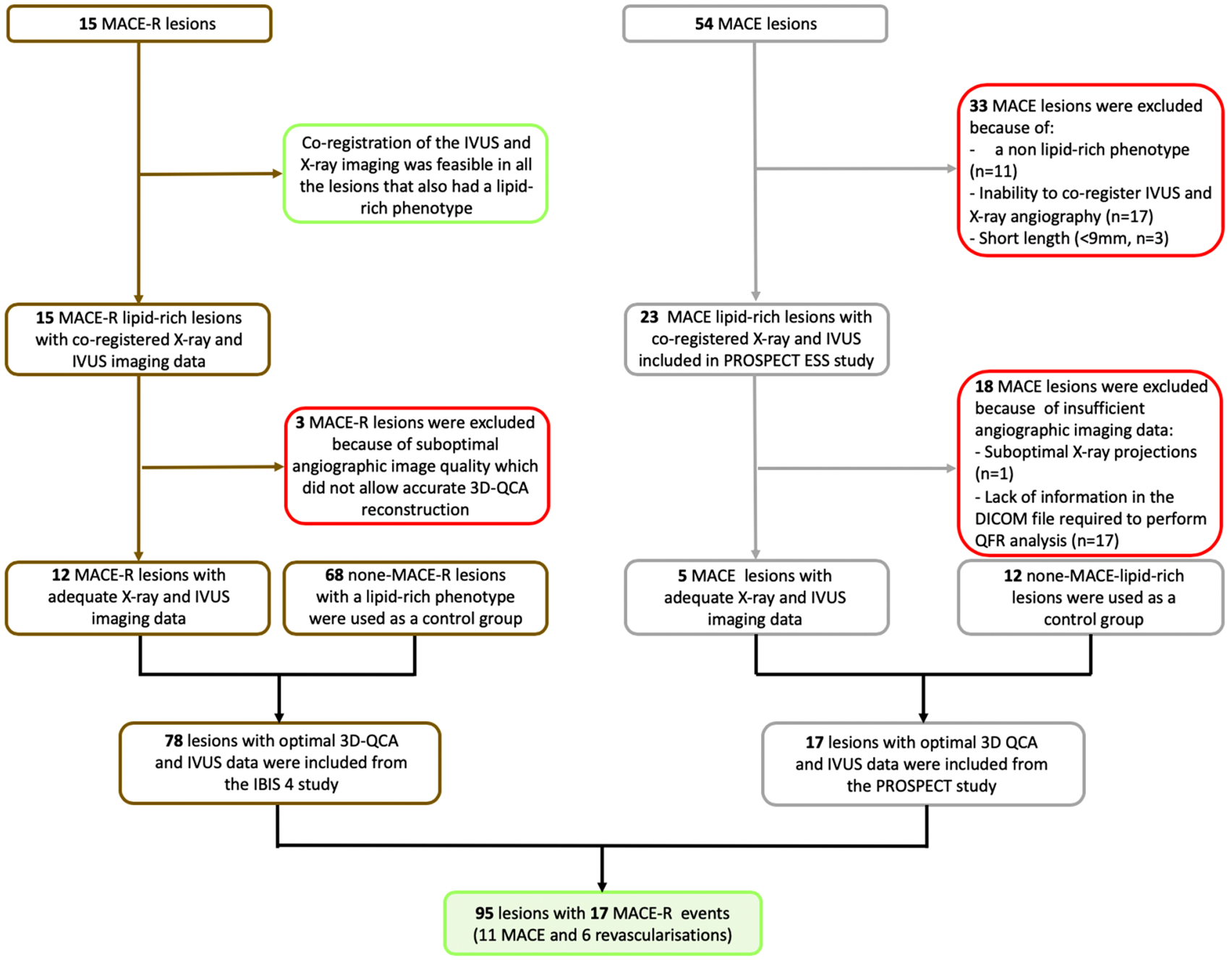

Fig. 1 Flowchart of study design

MACE lesions had a smaller MLA and greater PB on VH-IVUS compared to non-MACE lesions. On 3D-QCA analysis MACE lesions were longer than the non-MACE lesions and had increased \%DS but there were no differences in the MLD between groups. Both CQFR and fQFR were lower in the lesions that caused MACE.

\section{Predictors of MACE lesions}

Two VH-IVUS-derived variables (MLA and PB), two 3D-QCA-derived variables (lesion length and MLD) and the FQFR and CQFR indices were predictors of MACE in univariate analysis (Table 3 ). In ROC curve analysis PB (AUC: $0.751, \mathrm{P}=0.001$ ), MLA (AUC: $0.734, \mathrm{P}=0.003$ ) and cQFR (cutoff 0.97; AUC: $0.733, \mathrm{P}=0.003$ ) were the three variables that had the highest AUC for predicting MACE (AUC for fQFR: 0.716, P=0.005). In the multivariable analysis that included cQFR and the presence of lesions with PB $>70 \%$ and small MLA $<4.0 \mathrm{~mm}^{2}$, a small cQFR $(<0.97)$ was independently associated with future events (HR 3.53, 95\% CI 1.16-10.75; $\mathrm{P}=0.027$ ); in this model the presence of lesions with increased PB and small MLA was not an independent predictor of MACE (HR 1.87, 95\% CI 0.64-5.43; $\mathrm{P}=0.252$; Fig. 2).

Lesions were classified into 4 groups according to the presence of $\geq 2$ out of the 3 high-risk plaque characteristics that in the PROSPECT study were associated with MACE (MLA $\leq 4 \mathrm{~mm}^{2}, \mathrm{~PB} \geq 70 \%$, TCFA phenotype) and the CQFR value $(<0.97)$. Lesions with high-risk plaque characteristics and low cQFR had worse prognosis than the other lesions. Similar results were obtained when a high-risk plaque phenotype was defined as the presence of MLA $\leq 4 \mathrm{~mm}^{2}$ and $\mathrm{PB} \geq 70 \%$ (Fig. 3a, b). 
Table 1 Studied patients' baseline demographics

\begin{tabular}{|c|c|c|c|c|}
\hline & $\begin{array}{l}\text { Studied patients } \\
(\mathrm{N}=60)\end{array}$ & $\begin{array}{l}\text { Non-MACE group } \\
(\mathrm{N}=44)\end{array}$ & MACE group $(\mathrm{N}=16)$ & $\mathrm{P}$ \\
\hline Age (years) & $57.1 \pm 10.13$ & $56.8 \pm 10.4$ & $57.7 \pm 9.6$ & 0.707 \\
\hline Gender (male) & $55(92 \%)$ & $40(91 \%)$ & $15(94 \%)$ & 1.000 \\
\hline BMI & $27.7 \pm 4.2$ & $27.6 \pm 4.4$ & $27.9 \pm 3.8$ & 0.498 \\
\hline Current smoker & $29(48 \%)$ & $18(41 \%)$ & $11(68.8 \%)$ & 0.056 \\
\hline \multicolumn{5}{|l|}{ Co-morbidities } \\
\hline Diabetes mellitus & $8(13 \%)$ & $5(11 \%)$ & $3(19 \%)$ & 0.429 \\
\hline Hypertension & $27(45 \%)$ & $21(48 \%)$ & $6(38 \%)$ & 0.481 \\
\hline Hypercholesterolemia & $25(43 \%)$ & $20(46 \%)$ & $5(36 \%)$ & 0.522 \\
\hline Renal failure* & $2(4 \%)$ & $1(2 \%)$ & $1(8 \%)$ & 0.414 \\
\hline Previous PCI & $4(7 \%)$ & $3(7 \%)$ & $1(6 \%)$ & 1.000 \\
\hline Family history of CAD & $20(34 \%)$ & $15(34 \%)$ & $5(33 \%)$ & 0.957 \\
\hline Clinical presentation & & & & 0.739 \\
\hline STEMI & $51(85 \%)$ & $38(86 \%)$ & $13(81 \%)$ & \\
\hline NSTEMI & $7(12 \%)$ & $5(11 \%)$ & $2(13 \%)$ & \\
\hline Unstable angina & $2(3 \%)$ & $1(2 \%)$ & $1(6 \%)$ & \\
\hline \multicolumn{5}{|l|}{ Medications at discharge } \\
\hline Aspirin & $60(100 \%)$ & $44(100 \%)$ & $16(100 \%)$ & - \\
\hline Thienopyridines & $60(100 \%)$ & $44(100 \%)$ & $16(100 \%)$ & - \\
\hline Beta-blocker & $56(93 \%)$ & $42(96 \%)$ & $14(88 \%)$ & 0.287 \\
\hline RAAS inhibitor & $55(92 \%)$ & $42(96 \%)$ & $13(81 \%)$ & 0.112 \\
\hline Statin & $60(100 \%)$ & $44(100 \%)$ & $16(100 \%)$ & - \\
\hline
\end{tabular}

$B M I$ body mass index, $C A D$ coronary artery disease, $M A C E$ major adverse cardiovascular event, $N S T E M I$ non-ST-elevation myocardial infarction, $P C I$ percutaneous coronary intervention, $R A A S$ renin angiotensin aldosterone system, STEMI ST-elevation myocardial infarction

*Renal failure was defined as estimated glomerular filtration rate $<60 \mathrm{~mL} / \mathrm{min} / 1.73 \mathrm{~m}^{2}$

\section{Discussion}

In this study, we have for the first time examined the value of the computationally derived FFR estimated from the QFR software in identifying non-flow limiting lesions with a vulnerable-lipid-rich phenotype that are likely to progress and cause MACE within 5-year follow-up. We analysed data from two large-scale intravascular imaging studies and found that the QFR software provides additional prognostic information and that together with the plaque characteristics derived by VH-IVUS enables more accurate detection of the lesions associated with MACE-R.

Large scale prospective intravascular imaging studies of coronary atherosclerosis have demonstrated that an invasive assessment of plaque morphology allows detection of high-risk plaque features and identification of lesions that are likely to progress and cause events [9, 14-16]. However, in these studies the positive predictive value of the studied invasive imaging modalities in predicting MACE was low. Therefore their routine use to stratify risk in the clinical setting is currently not recommended [17]. Combination of plaque morphology and physiology, particularly estimation of the ESS using computational fluid dynamic analysis of intravascular imaging data seems to provide additional prognostic information and detection of vulnerable plaques with a positive predictive value that exceeds $>50 \%[11,18]$. However, ESS computation is a time consuming process that requires dedicated software and expertise, facts that limit its broad use in clinical practice.

FFR was introduced to assess in real time the physiologic implications of coronary lesions and detect those that cause flow obstruction and ischemia. Reports have shown an inverse association between baseline FFR and the incidence of future events, highlighting the prognostic value of this metric, even in non-flow limiting lesions [19, 20]. Studies also support a high agreement between FFR and QFR in patients with an acute coronary syndrome and underscore the value of QFR in detecting lesions that are likely to progress and cause events [21, 22]. The prognostic value of QFR/FFR is also supported by reports comparing their estimations with IVUS imaging variables, these studies show a weak but statistically significant correlation between MLA or PB and the FFR values. Moreover, the COMPETE-OCT sub-study and a recent report that used multimodality intravascular imaging to assess plaque morphology demonstrated that significant stenoses are more likely to have a vulnerable phenotype $[23,24]$. These findings indicate that there is an association between low FFR values and high-risk plaque 
Table 2 Intravascular imaging, angiographic and hemodynamic characteristics of the studied lesions

\begin{tabular}{|c|c|c|c|}
\hline & $\begin{array}{l}\text { MACE-R lesions } \\
(\mathrm{N}=17)\end{array}$ & Non-MACE-R lesions $(\mathrm{N}=78)$ & $\mathrm{P}$ \\
\hline \multicolumn{4}{|l|}{ IVUS-VH plaque characteristics } \\
\hline Lesion length (mm) & $23.8(12.6,34.6)$ & $26.4(19.2,38.0)$ & 0.313 \\
\hline $\operatorname{MLA}\left(\mathrm{mm}^{2}\right)$ & $3.59(3.16,4.51)$ & $5.04(3.77,6.52)$ & 0.003 \\
\hline EEM area $\left(\mathrm{mm}^{2}\right)$ & $11.62(10.87,14.66)$ & $13.67(10.79,16.15)$ & 0.557 \\
\hline Plaque area $\left(\mathrm{mm}^{2}\right)$ & $8.69(7.10,10.51)$ & $7.83(6.57,10.72)$ & 0.449 \\
\hline $\mathrm{PB}(\%)$ & $70.4(63.5,72.2)$ & $61.0(53.3,67.6)$ & 0.001 \\
\hline TCFA phenotype & $12(71 \%)$ & $61(78 \%)$ & 0.500 \\
\hline \multicolumn{4}{|l|}{ QCA derived variables } \\
\hline Lesion length & $16.6(10.7,20.6)$ & $9.3(6.2,14.5)$ & 0.008 \\
\hline MLD & $1.90(1.60,2.10)$ & $2.00(1.70,2.40)$ & 0.192 \\
\hline Diameter stenosis & $35.0(30.6,41.1)$ & $28.7(23.7,39.0)$ & 0.029 \\
\hline \multicolumn{4}{|l|}{ QFR derived variables } \\
\hline Fixed QFR & $0.95(0.93,0.98)$ & $0.99(0.96,1.00)$ & 0.005 \\
\hline Contrast FFR & $0.94(0.90,0.98)$ & $0.99(0.95,1.00)$ & 0.002 \\
\hline \multicolumn{4}{|l|}{ Lesion location } \\
\hline Coronary artery & & & 0.278 \\
\hline Left anterior descending artery & $9(53 \%)$ & $33(42 \%)$ & \\
\hline Left circumflex artery & $7(41 \%)$ & $23(30 \%)$ & \\
\hline Right coronary artery & $1(6 \%)$ & $21(27 \%)$ & \\
\hline Intermediate coronary artery & $0(0 \%)$ & $1(1 \%)$ & \\
\hline Coronary segment & & & 0.965 \\
\hline Proximal vessel & $13(76 \%)$ & $59(76 \%)$ & \\
\hline Mid vessel & $3(18 \%)$ & $13(17 \%)$ & \\
\hline Distal vessel & $1(6 \%)$ & $6(8 \%)$ & \\
\hline
\end{tabular}

Table 3 Univariable analyses of the IVUS, QCA-derived predictors of MACE-R lesions

\begin{tabular}{lll}
\hline & \multicolumn{2}{l}{ Univariable analysis } \\
\cline { 2 - 3 } & Hazard ratio & $\mathrm{P}$ \\
\hline IVUS-derived variables & & \\
MLA (per 1 mm ${ }^{2}$ increase) & $0.51(0.32,0.79)$ & 0.003 \\
PB (per 1\% increase) & $1.11(1.05,1.18)$ & 0.001 \\
QCA-derived variables & & \\
Lesion length (per 1 mm increase) & $1.10(1.01,1.09)$ & 0.010 \\
MLD (per 1 mm increase) & $0.34(0.12,0.92)$ & 0.033 \\
fQFR (per 0.1 unit increase) & $0.36(0.16,0.81)$ & 0.013 \\
cQFR (per 0.1 increase) & $0.43(0.21,0.89)$ & 0.022 \\
\hline
\end{tabular}

features (i.e., MLA, PB and a vulnerable phenotype) that are predictors of future events.

The present study examined for the first time the combined value of computationally-derived FFR and plaque morphology in predicting events. We analysed data from the PROSPECT and IBIS-4 studies and used an established software that is capable of retrospectively processing angiographic imaging data to derive FFR [7]. We found that QFR provided additional prognostic information to plaque morphology and enabled more accurate prediction of MACE. In particular $40.4 \%$ of the lesions with high risk plaque characteristics and low QFR values caused MACE while the event rate was lower in lesions with QFR $\geq 0.96$ or a non-high risk plaque morphology. These findings highlight the potential importance of the combined assessment of plaque morphology and physiology for more accurate characterisation of plaque vulnerability. Future prospective studies or retrospective analyses of currently ongoing intravascular imaging studies of atherosclerosis should be performed to validate the findings from the present report. Such studies may provide insight about the predictive efficacy of this approach in detecting vulnerable plaques and high-risk patients who might benefit from an aggressive treatment of atherosclerosis [25, 26].

Advances in image processing and computational methods are anticipated to facilitate research towards this direction. Several solutions have been proposed in the literature for intravascular imaging analysis and fast computation of the pressure gradient across a lesion at rest or during hyperemia [27-29]. The OFR software is the first userfriendly tool that enables real-time segmentation of OCT data and evaluation of lesion severity using computational modelling. Validation of this approach has shown a high 

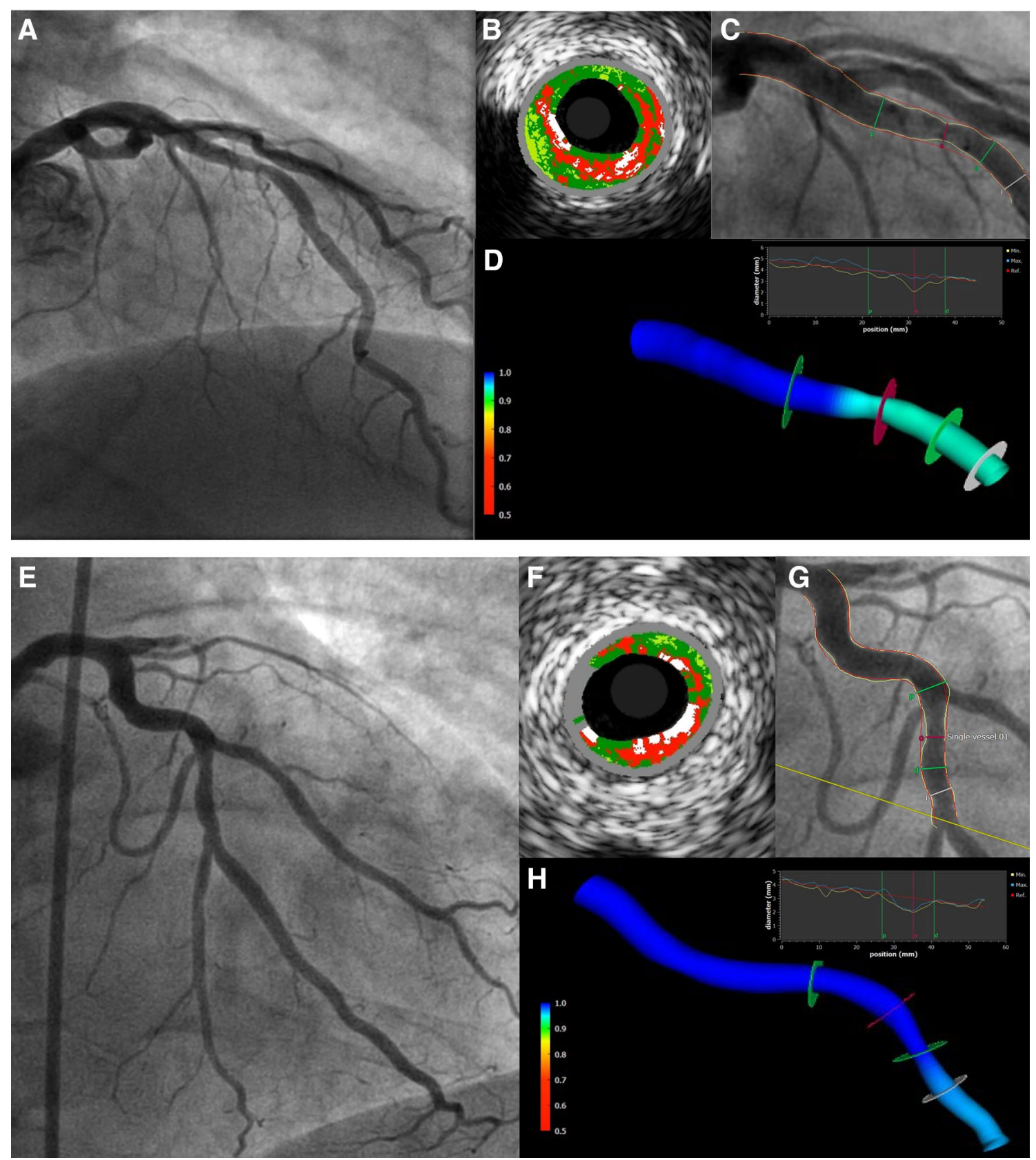

Fig. 2 Case examples that underscore the additional value of QFR in predicting lesions associated with MACE-R. a Shows the angiographic projection of a lesion that progressed and required revascularization at 1-year follow-up. VH-IVUS assessment of this lesion demonstrated a ThCFA phenotype with a MLA of $4.11 \mathrm{~mm}^{2}$ and a $\mathrm{PB}$ of $71.7 \%$ (b). c Shows the QCA analysis of the segment assessed by VH-IVHS which indicates a minor stenosis (MLD: $2.0 \mathrm{~mm}$, DS: 42.0\%), while (d) the estimated QFR values (fQFR: 0.94, cQFR:

agreement between OFR and FFR estimations [30, 31]. Similar software are expected to be designed in the future for the analysis of IVUS imaging data and used to predict more accurately lesions that are likely to progress and cause events compared to standalone imaging.
0.94). e Portrays the angiographic projection of a lesion that remained quiescent. VH-IVUS examination demonstrated a TCFA phenotype with a MLA of $3.77 \mathrm{~mm}^{2}$ and PB of $60.9 \%$ (f) while in the QCA analysis, the estimated MLD and \%DS were similar to the previous lesion (MLD: $2.0 \mathrm{~mm}$, \%DS: 34.5) (g). In this occasion however, the QFR values were higher (fQFR: 0.98, cQFR: 0.97) than the lesion that progressed and required revascularisation (h).

\section{Study limitations}

The key limitations of the present retrospective study is the fact that a large proportion of lesions were excluded from the analysis. This is because either accurate co-registration of 


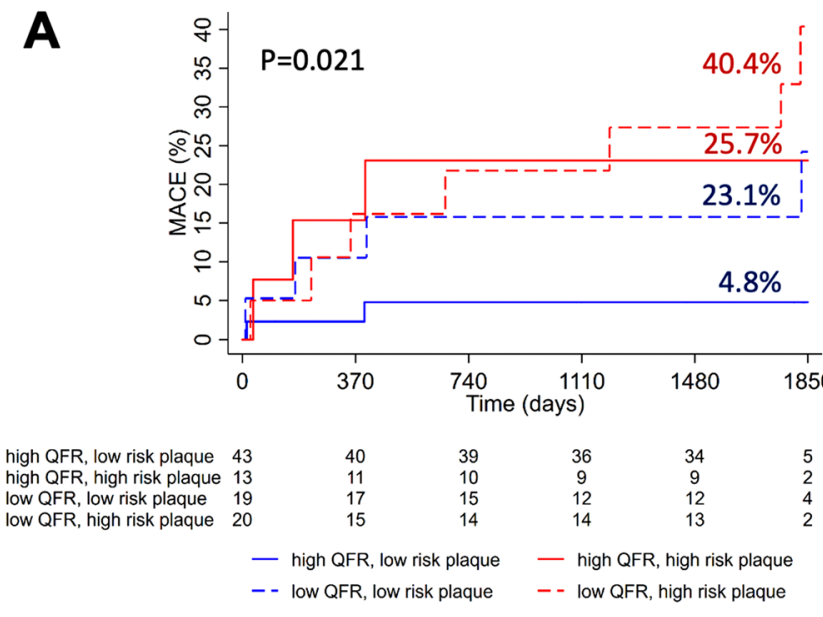

Fig. 3 Kaplan-Meier curves showing the MACE-R rate in lesions classified according to VH-IVUS and QFR metrics. Lesions were classified into groups according to the presence or absence of (a) high-risk plaque morphology (i.e., plaques with $\geq 2$ high-risk fea-

IVUS and X-ray imaging was not possible or/and the DICOM information required to estimate QFR was not available in the angiograms acquired in the PROSPECT study. In addition, QFR analysis was performed in angiographic data that had already been acquired; a fact that may have affected the accuracy of QFR in assessing the hemodynamic severity of a lesion. Moreover, the number of events reported was small and thus in order to avoid model overfitting we included in the multivariate model only 2 non-colinear variables-presence of $\mathrm{PB}>70 \%$ and $\mathrm{MLA}<4 \mathrm{~mm}^{2}$ and QFR—out of the 6 variables that were associated with MACE in the univariate analysis. Therefore, this analysis should be considered as an exploratory and hypothesis generating analysis requiring validation from larger studies. Moreover, VH-IVUS imaging was carried out using two different imaging systems (s5 in IBIS-4 and Invision Gold in PROSPECT); VH assessment from these two systems could give different estimations for plaque composition but we believe that it will have less effect in the estimation of plaque phenotype. In addition, the analysis of the VH-IVUS data was performed by two different core-labs, however they are in close collaboration and use the same classification algorithm to define plaque phenotype [12]. Finally, the follow-up period (median 3.4 years in PROSPECT and 5 years in IBIS-4) and the clinical end-points were different in the two studies as the IBIS-4 study also reported revascularizations because of disease progression in coronary angiography and established evidence of ischemia at 13 months follow-up.

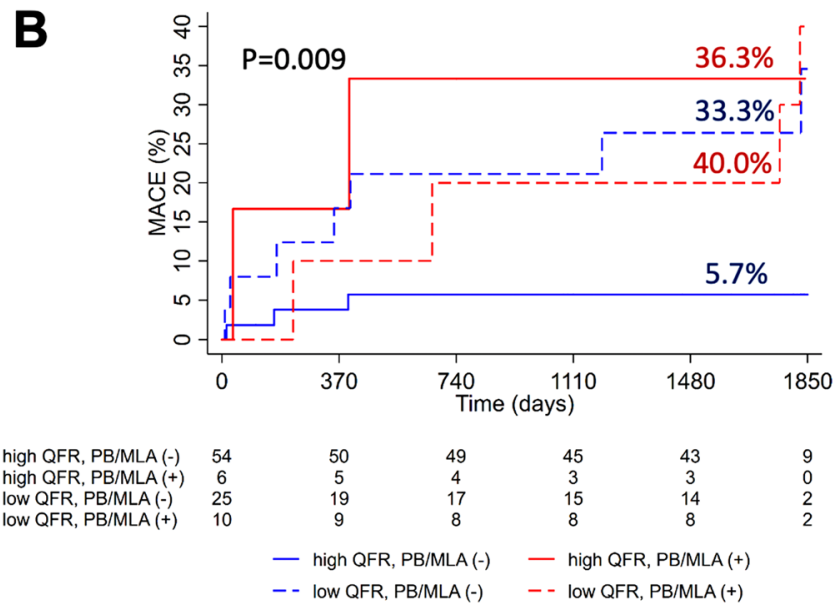

tures: defined as MLA $<4 \mathrm{~mm}^{2}, \mathrm{~PB}>70 \%$ and a TCFA phenotype) and a low cQFR value $(<0.97)$ and of $(\mathbf{b}) \mathrm{PB}>70 \%$ and $\mathrm{MLA}<4 \mathrm{~mm}^{2}$ and low cQFR value

\section{Conclusions}

In the present post-hoc analysis, assessment of plaque physiology using commercially available QFR software appears to provide additional prognostic information and more accurate identification of lesions that are likely to progress and cause MACE at 5-year follow-up compared to VH-IVUS derived plaque morphology alone. However, the small number of events reported do not allow us to draw firm conclusions. Therefore, further confirmatory research is needed in a larger number of patients to quantify the predictive accuracy of combined QFR and intravascular imaging for the identification of vulnerable plaques and vulnerable patients.

Acknowledgements HS is funded by research funds of the British Heart Foundation (Project Grant: PG/17/18/32883), TZ by the Swiss National Science Foundation (Grant Number: 323530-171146), AR by research funds of Whipps Cross University Hospital while AB, AM and CB are supported by the Barts NIHR Biomedical Research Centre.

Author contributions All authors take responsibility for all aspects of the reliability and freedom from bias of the data presented and their discussed interpretation.

\section{Compliance with ethical standards}

Conflict of interest PWS, GWS and AK have received personal fees from Philips/Volcano and Abbott Vascular, CVB from Philips/Volcano, SW from Abbott and LR from Abbott, Amgen, AstraZeneca, 
CSL Behring, Sanofi, and Vifor and institutional fees from Abbott, Biotronik, Boston Scientific, Heartflow, Sanofi and Regeneron. JHCR is the CEO of Medis Medical Imaging Systems. None of the other authors have a conflict of interest to declare.

\section{References}

1. Park J, Lee JM, Koo BK et al (2018) Clinical relevance of functionally insignificant moderate coronary artery stenosis assessed by 3-vessel fractional flow reserve measurement. J Am Heart Assoc. https://doi.org/10.1161/JAHA.117.008055

2. Ahmed N, Layland J, Carrick D et al (2016) Safety of guidewirebased measurement of fractional flow reserve and the index of microvascular resistance using intravenous adenosine in patients with acute or recent myocardial infarction. Int J Cardiol. https:// doi.org/10.1016/j.ijcard.2015.09.014

3. Tu S, Westra J, Yang J et al (2016) Diagnostic accuracy of fast computational approaches to derive fractional flow reserve from diagnostic coronary angiography: the international multicenter FAVOR Pilot Study. JACC Cardiovasc Interv. https://doi. org/10.1016/j.jcin.2016.07.013

4. Westra J, Andersen BK, Campo G et al (2018) Diagnostic performance of in-procedure angiography-derived quantitative flow reserve compared to pressure-derived fractional flow reserve: the FAVOR II Europe-Japan Study. J Am Heart Assoc. https://doi. org/10.1161/JAHA.118.009603

5. Xu B, Tu S, Qiao S et al (2017) Diagnostic accuracy of angiography-based quantitative flow ratio measurements for online assessment of coronary stenosis. J Am Coll Cardiol. https://doi. org/10.1016/j.jacc.2017.10.035

6. Hamaya R, Hoshino M, Kanno Y et al (2019) Prognostic implication of three-vessel contrast-flow quantitative flow ratio in patients with stable coronary artery disease. EuroIntervention 15:180-188. https://doi.org/10.4244/EIJ-D-18-00896

7. Tu S, Westra J, Adjedj J et al (2019) Fractional flow reserve in clinical practice: from wire-based invasive measurement to imagebased computation. Eur Heart J. https://doi.org/10.1093/eurheartj/ ehz918

8. Lee JM, Choi G, Koo BK et al (2018) Identification of high-risk plaques destined to cause acute coronary syndrome using coronary computed tomographic angiography and computational fluid dynamics. JACC Cardiovasc Imaging. https://doi.org/10.1016/j. jcmg.2018.01.023

9. Stone GW, Maehara A, Lansky AJ et al (2011) A prospective Natural-History Study of coronary atherosclerosis. N Engl J Med. https://doi.org/10.1056/NEJMoa1002358

10. Räber L, Taniwaki M, Zaugg S et al (2015) Effect of high-intensity statin therapy on atherosclerosis in non-infarct-related coronary arteries (IBIS-4): a serial intravascular ultrasonography study. Eur Heart J 36:490-500. https://doi.org/10.1093/eurheartj/ehu373

11. Stone PH, Maehara A, Coskun AU et al (2018) Role of low endothelial shear stress and plaque characteristics in the prediction of nonculprit major adverse cardiac events: the PROSPECT Study. JACC Cardiovasc Imaging. https://doi.org/10.1016/j. jemg.2017.01.031

12. García-García HM, Mintz GS, Lerman A et al (2009) Tissue characterisation using intravascular radiofrequency data analysis: Recommendations for acquisition, analysis, interpretation and reporting. EuroIntervention. https://doi.org/10.4244/EIJV5I2A29

13. Tu S, Huang Z, Koning G et al (2010) A novel three-dimensional quantitative coronary angiography system: In-vivo comparison with intravascular ultrasound for assessing arterial segment length. Catheter Cardiovasc Interv. https://doi.org/10.1002/ ccd.22502
14. Calvert PA, Obaid DR, O'Sullivan M et al (2011) Association between IVUS findings and adverse outcomes in patients with coronary artery disease: the VIVA (VH-IVUS in vulnerable atherosclerosis) study. JACC Cardiovasc Imaging. https://doi. org/10.1016/j.jcmg.2011.05.005

15. Waksman R (2018) Assessment of coronary near-infrared spectroscopy imaging to detect vulnerable plaques and vulnerable patients. The Lipid-Rich Plaque study. San Diego, pp 21-25

16. Prati F, Romagnoli E, Gatto L et al (2018) Relationship between OCT coronary plaque morphology and clinical outcome (CLIMA). In: EuroPCR, Paris

17. Johnson TW, Räber L, di Mario C et al (2019) Clinical use of intracoronary imaging. Part 2: acute coronary syndromes, ambiguous coronary angiography findings, and guiding interventional decision-making: an expert consensus document of the European Association of Percutaneous Cardiovascular Intervent. Eur Heart J. https://doi.org/10.1093/eurheartj/ehz332

18. Papafaklis MI, Mizuno S, Takahashi S et al (2016) Incremental predictive value of combined endothelial shear stress, plaque necrotic core, and plaque burden for future cardiac events: a posthoc analysis of the PREDICTION Study. Int J Cardiol. https://doi. org/10.1016/j.ijcard.2015.08.208

19. Barbato E, Toth GG, Johnson NP et al (2016) A prospective Natural History Study of Coronary atherosclerosis using fractional flow reserve. J Am Coll Cardiol. https://doi.org/10.1016/j. jacc.2016.08.055

20. Johnson NP, Tóth GG, Lai D et al (2014) Prognostic value of fractional flow reserve: linking physiologic severity to clinical outcomes. J Am Coll Cardiol. https://doi.org/10.1016/j. jacc.2014.07.973

21. Spitaleri G, Tebaldi M, Biscaglia S et al (2018) Quantitative flow ratio identifies Nonculprit Coronary lesions requiring revascularization in patients with ST-segment-elevation myocardial infarction and multivessel disease. Circ Cardiovasc Interv. https://doi. org/10.1161/CIRCINTERVENTIONS.117.006023

22. Lauri F, Macaya F, Mejía-Rentería H et al (2019) Angiographyderived functional assessment of non-culprit coronary stenoses during primary percutaneous coronary intervention for STelevation myocardial infarction. EuroIntervention. https://doi. org/10.4244/EIJ-D-18-01165

23. Pinilla-Echeverri N, Mehta SR, Wang J et al (2019) Non-culprit lesion plaque morphology in patients with ST-Segment elevation myocardial infarction: results from the COMPLETE trial optical coherence tomography (OCT) Substudy. American Heart Association Scientific Sessions, Philadelphia

24. Tian J, Dauerman H, Toma C et al (2014) Prevalence and characteristics of TCFA and degree of coronary artery stenosis: An OCT, IVUS, and angiographic study. J Am Coll Cardiol. https:// doi.org/10.1016/j.jacc.2014.05.052

25. Bourantas CV, Garcia-Garcia HM, Diletti R et al (2013) Early detection and invasive passivation of future culprit lesions: a future potential or an unrealistic pursuit of chimeras? Am Heart J 165:869-881

26. Bourantas CV, Garcia-Garcia HM, Torii R et al (2016) Vulnerable plaque detection: an unrealistic quest or a feasible objective with a clinical value? Heart 102:581-589

27. Ha J, Kim JS, Lim J et al (2016) Assessing computational fractional flow reserve from optical coherence tomography in patients with intermediate coronary stenosis in the left anterior descending artery. Circ Cardiovasc Interv. https://doi.org/10.1161/CIRCI NTERVENTIONS.116.003613

28. Jang SJ, Ahn JM, Kim B et al (2017) Comparison of accuracy of one-use methods for calculating fractional flow reserve by intravascular optical coherence tomography to that determined by the pressure-wire method. Am J Cardiol. https://doi.org/10.1016/j. amjcard.2017.08.010 
29. Siogkas PK, Papafaklis MI, Lakkas L et al (2019) Virtual functional assessment of coronary stenoses using intravascular ultrasound imaging: a proof-of-concept Pilot Study. Heart Lung Circ. https://doi.org/10.1016/j.hlc.2018.02.011

30. Yu W, Huang J, Jia D et al (2019) Diagnostic accuracy of intracoronary optical coherence tomography-derived fractional flow reserve for assessment of coronary stenosis severity. EuroIntervention. https://doi.org/10.4244/eij-d-19-00182

31. Huang J, Emori H, Ding D et al (2020) Comparison of diagnostic performance of intracoronary optical coherence tomography-based and angiography-based fractional flow reserve for evaluation of coronary stenosis. EuroIntervention. https://doi. org/10.4244/EIJ-D-19-01034

Publisher's Note Springer Nature remains neutral with regard to jurisdictional claims in published maps and institutional affiliations. 